\title{
MANET performance for source and destination moving scenarios considering OLSR and AODV protocols
}

\author{
Elis Kulla ${ }^{\mathrm{a}, *}$, Masahiro Hiyama ${ }^{\mathrm{a}}$, Makoto Ikeda ${ }^{\mathrm{b}}$, Leonard Barolli ${ }^{\mathrm{c}}$, Vladi Kolici ${ }^{\mathrm{d}}$ and \\ Rozeta Miho ${ }^{\mathrm{d}}$ \\ ${ }^{\mathrm{a}}$ Graduate School of Engineering, Fukuoka Institute of Technology (FIT), 3-30-1 Wajiro-Higashi, \\ Higashi-Ku, Fukuoka, Japan \\ ${ }^{\mathrm{b}}$ Center for Asian and Pacific Studies, Seikei University, 3-3-1 Kichijoji-Kitamachi, Musashino-Shi, \\ Tokyo, Japan \\ ${ }^{\mathrm{c}}$ Department of Information and Communication Engineering, Fukuoka Institute of Technology (FIT), \\ 3-30-1 Wajiro-Higashi, Higashi-Ku, Fukuoka, Japan \\ ${ }^{\mathrm{d}}$ Department of Electronic and Telecommunication, Polytechnic University of Tirana, Mother Teresa \\ Square, Nr. 4, Tirana, Albania
}

\begin{abstract}
Recently, a great interest is shown in MANETs potential usage and applications in several fields such as military activities, rescue operations and time-critical applications. In this work, we implement and analyse a MANET testbed considering AODV and OLSR protocols for wireless multi-hop networking. We investigate the effect of mobility and topology changing in MANET and evaluate the performance of the network through experiments in a real environment. The performance assessment of our testbed is done considering throughput, number of dropped packets and delay. We designed four scenarios: Static, Source Moving, Destination Moving and Source-Destination Moving. From our experimental results, we concluded that when the communicating nodes are moving and the routes change quickly, OLSR (as a proactive protocol) performs better than AODV, which is a reactive protocol.
\end{abstract}

\section{Introduction}

During recent years, we have witnessed a lot of research on wireless networks [5,16,17,21,1,8,24,25]. There are two network architectures for wireless networks: infrastructure and ad-hoc architecture.

Wireless networks often extend, rather than replace, wired networks, which are referred to as infrastructure networks. The wide area and local area wired networks are used as the backbone network. The wired backbone connects to special switching nodes called Base Stations (BSs). The BSs are often conventional PCs and workstations equipped with custom wireless adapter cards. They are responsible for coordinating access to one or more transmission channel(s) for mobiles located within the coverage cell.

Ad-hoc networks, on the other hand, are multi-hop wireless networks in which a set of mobile nodes cooperatively maintain network connectivity. Ad-hoc networks are characterized by dynamic,

\footnotetext{
${ }^{*}$ Corresponding author. E-mail: eliskulla@yahoo.com.
} 
unpredictable, random, multi-hop topologies with typically no infrastructure support. The mobile nodes must periodically exchange topology information which is used for routing updates.

A Mobile Ad hoc Network (MANET) is a collection of wireless mobile terminals that are able to dynamically form a temporary network without any aid from fixed infrastructure or centralized administration. In recent years, MANET are continuing to attract the attention for their potential use in several fields. Mobility and the absence of any fixed infrastructure make MANET very attractive for mobility and rescue operations and time-critical applications.

Most of the work for MANETs has been done in simulation, as in general, a simulator can give a quick and inexpensive understanding of protocols and algorithms $[9,10,20,26]$. However, experimentation in the real world are very important to verify the simulation results and to revise the models implemented in the simulator. A typical example of this approach has revealed many aspects of IEEE 802.11, like the gray-zones effect [14], which usually are not taken into account in standard simulators, as the well-known $n s-2$ simulator [22].

So far, we can count a lot of computer simulation results on the performance of MANET, e.g. in terms of end-to-end throughput, delay and packet loss. However, in order to assess the computer simulation results, real-world experiments are needed and a lot of testbeds have been built to date $[12,23,13]$. The baseline criteria usually used in real-world experiments is guaranteeing the repeatability of tests, i.e. if the system does not change along the experiments. How to define a change in the system is not a trivial problem in MANET, especially if the nodes are mobile.

In this paper, we focus on comparing the performance of two types of routing protocols Ad-hoc On demand Distance Vector (AODV), which is a reactive routing protocol, and Optimized Link State Routing (OLSR), which is a proactive routing protocol. Both protocols have been gaining great attention within the scientific community. Furthermore, the $a o d v-u u$ [4] and the olsrd [18] software we have used in our experiments are the most updated software we have encountered.

In our previous work, we found the following results. We proved that while some of the OLSR's problems can be solved, for instance the routing loop, this protocol still have the self-interference problem. There is an intricate inter-dependence between MAC layer and routing layer, which can lead the experimenter to misunderstand the results of the experiments. We carried out the experiments considering stationary nodes of ad-hoc network. We considered the node mobility and carry out experiments for AODV, OLSR and BATMAN protocols [2]. We found that throughput of TCP were improved by reducing Link Quality Window Size (LQWS), but there were packet loss because of experimental environment and traffic interference. For TCP data flow, we got better results when the LQWS value was 10.

In this work, we implemented four MANET scenarios and carried out real world experiments in an indoor environment. We assess the performance of two routing protocols AODV and OLSR for different source and destination moving scenarios.

The structure of the paper is as follows. In Section 2, we give a short description of AODV and OLSR. In Section 3, we describe the testbed and its implementation. The moving scenarios are described in Section 4. In Section 5, we present experimental evaluation. Finally, conclusions are given in Section 6.

\section{Routing protocols}

\subsection{AODV overview}

AODV is one of the most popular reactive routing protocol for MANETs [19]. As a reactive (on demand) protocol, when a node wants to transmit data, it first starts a route discovery process, by 
flooding a RREQ (Route Request) packet. The RREQ packets are forwarded by all nodes by which they are received. This procedure continues until the destination is found. On the way to destination, the RREQ informs all the intermediate nodes about a route to the source. When the RREQ reaches the destination, destination sends a Route Reply (RREP) packet which follows the reverse path discovered by RREQ. This informs all intermediate nodes about a route to the destination node. After RREQ and RREP are delivered to their destination, each intermediate node on the route knows what node to forward data packets in order to reach source or destination. Thus data packets do not need to carry addresses of all intermediate nodes in the route. It just carries the address of the destination node, decreasing noticeably routing overheads.

A third kind of routing message, called Route Error (RERR), allows nodes to notify errors, for example, because a previous neighbor has moved and is no longer reachable. If the route is not active (i.e., there is no data traffic flowing through it), all routing information expire after a timeout and is removed from the routing table.

In AODV, the route discovery process may last for a long time, or it can be repeated several times, due to potential failures during the process. This introduces extra delays, and consumes more bandwidth as the size of the network increases.

\subsection{OLSR overview}

The link state routing protocol that is most popular today in the open source world is OLSR from olsr.org. OLSR with Link Quality (LQ) extension and fisheye-algorithm works quite well. The OLSR protocol is a pro-active routing protocol, which builds up a route for data transmission by maintaining a routing table inside every node of the network.

The routing table is computed upon the knowledge of topology information, which is exchanged by means of Topology Control (TC) packets. The TC packets in turn are built after every node has filled its neighbors list. This list contains the identity of neighbor nodes. A node is considered a neighbor if and only if it can be reached via a bi-directional link. OLSR checks the symmetry of neighbors by means of a 4-way handshake based on the so called HELLO messages. This handshake is inherently used to compute the packet loss probability over a certain link. This can sound odd, because packet loss is generally computed at higher layer than routing one. However, an estimate of the packet loss is needed by OLSR in order to assign a weight or a state to every link.

In OLSR, control packets are flooded within the network by electing special nodes, called Multi Point Relays (MPRs), to the role of forwarding nodes. By this way, the amount of control traffic can be reduced. These nodes are chosen in such a way that every node can reach its neighbors 2-hops far away. In our OLSR code, a simple RFC-compliant heuristic is used [3] to compute the MPR nodes. Every node computes the path towards a destination by means of a simple shortest-path algorithm, with hop-count as target metric. In this way, a shortest path can result to be also not good, from the point of view of the packet error rate. Accordingly, recently OLSRd has been equipped with the Link Quality (LQ) extension, which is a shortest-path algorithm with the average of the packet error rate as metric. This metric is commonly called as the Expected Transmission Rate (ETX), which is defined as $\operatorname{ETX}(i)=1 /(N I(i) \times L Q I(i))$. Given a sampling window $W, \mathrm{NI}(i)$ is the packet loss probability seen by a node on the $i$-th link during $W$. Similarly, LQI $(i)$ is the estimation of the packet loss seen by the neighbor node which uses the $i$-th link. When the link has a low packet error rate, the ETX metric is higher. The LQ extension greatly enhances the packet delivery ratio with respect to the hysteresis-based technique [6]. 
Table 1

Node addressing table

\begin{tabular}{ccl}
\hline Node ID & IP Address & Operating System \\
\hline Node 1 & 192.168 .0 .1 & Fedora Core 4 \\
Node 2 & 192.168 .0 .2 & Ubuntu 9.04 \\
Node 3 & 192.168 .0 .5 & Ubuntu 9.04 \\
Node 4 & 192.168 .0 .6 & eeeUbuntu 9.04 \\
Node 5 & 192.168 .0 .7 & Ubuntu 9.04 \\
Node 6 & 192.168 .0 .10 & Ubuntu 9.04 \\
Node 7 & 192.168 .0 .11 & Ubuntu 9.04 \\
\hline
\end{tabular}

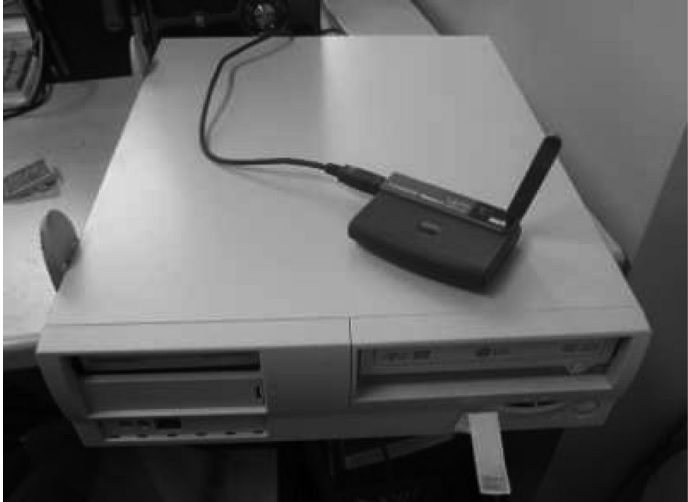

(a) Node 1

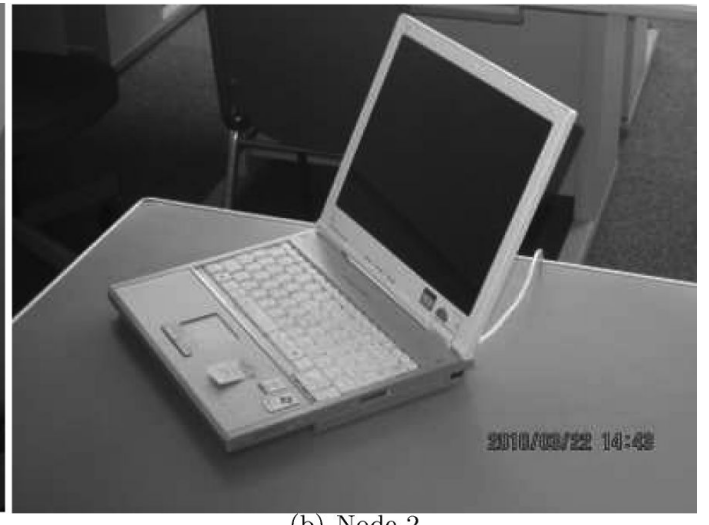

(b) Node 2

Fig. 1. Hardware of the testbed.

\section{Testbed description}

\subsection{Testbed environment}

We implemented a MANET testbed and carried out experiments in the fifth floor of Building D, at Fukuoka Institute of Technology. This testbed provides the environment to make different measurements for indoor and outdoor communications. However, in this paper we deal only with indoor environment.

\subsection{Operating system and routing software}

The operating system installed on machines is Ubuntu 9.04 Linux (x5), eeeUbuntu 9.04 Linux (x1) all with kernel 2.6.28-18-generic and Fedora Core 4 Linux (x1) as shown in Table 1. Each of them can support all installed routing softwares.

In each machine, the AODV and OLSR routing softwares are installed from their source code in their respective web pages. Both of them are open source. See $[4,18]$ for more information.

\subsubsection{Network configuration}

All machines used their own wireless adapter, except for the Fedora machine which uses a Linksys wireless card, whose drivers can be found at [15]. Each machine wireless card transmits at frequency $2.412 \mathrm{GHz}$ (channel 1), and is put to ad-hoc infrastructure. In Fig. 1, we show a screen-shot of every node we used in experiments. Node IDs and IP addresses are shown in Table 1. 


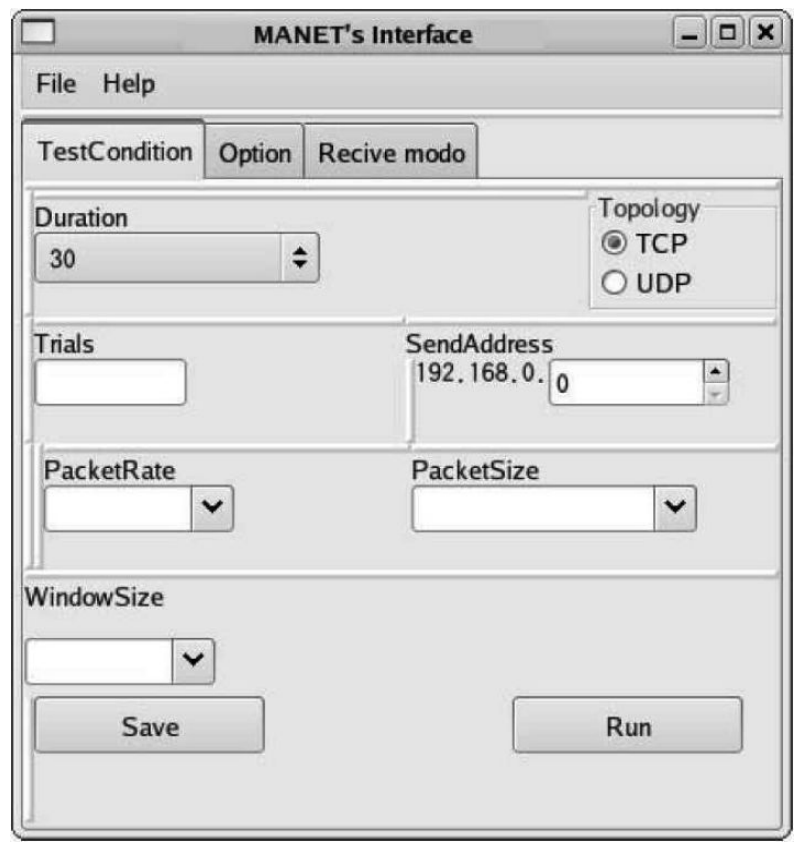

Fig. 2. GUI interface for parameter settings.

\subsubsection{Traffic generation and getting the data}

After configuring the network all nodes are put to their respective position, in accordance to the experimental scenario. To generate some traffic between nodes, we used D-ITG (Distributed Internet Traffic Generator) software, which is a Traffic Generator [7]. With D-ITG, one could send different type of traffics from one node to another. The amount of information to be sent and the duration of the transmission is set as an option. After finishing the transmission, D-ITG offers decoding tools to get information about network metrics along the whole transmission duration.

\subsubsection{Testbed interface}

All settings, editing and calculations can be done with the aid of a Graphical User Interface (GUI) as shown in [11]. This is helpful in saving time in the case of repeated experiments, and avoiding misprints during set-up. The GUI uses wxWidgets tool and each operation is implemented by Perl language. wxWidgets is a cross-platform GUI and tools library for GTK, MS Windows and Mac OS X. Many parameters are implemented in the interface such as transmission duration, number of trials, source address, destination address, packet rate, packet size, LQWS, and topology setting function. These parameters can be saved in a text file and can manage the experimental conditions in a better approach. The GUI interface of the implemented testbed is shown in Fig. 2.

\section{Topology description}

The implemented testbed provides a real-time system for analysing various aspects of MANETs. The purpose of this paper is to evaluate the performance of two routing protocols: AODV and OLSR. Performance evaluation is done for four different scenarios. The MAC filtering is not used in these experiments, so the nodes form e Mesh Topology. We describe the four scenarios in the following. The 
Table 2

Experimental parameters

\begin{tabular}{lrrrc}
\hline Parameters & SS & SMS & DMS & SDMS \\
\hline Nr. of experiments & 20 & 10 & 10 & 10 \\
Duration of experiment(s) & 60 & 120 & 120 & 120 \\
Packet rate (pkt/s) & 200 & 200 & 200 & 200 \\
Packet size (bytes) & 512 & 512 & 512 & 512 \\
\hline
\end{tabular}

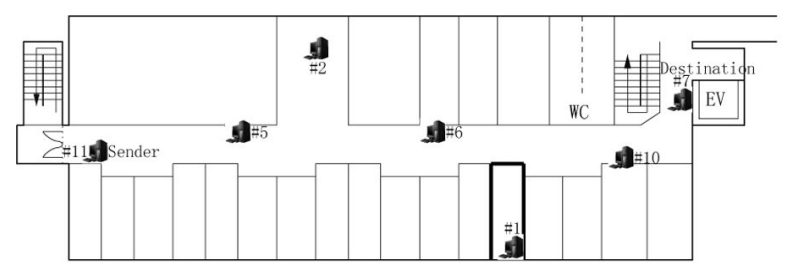

(a) SS

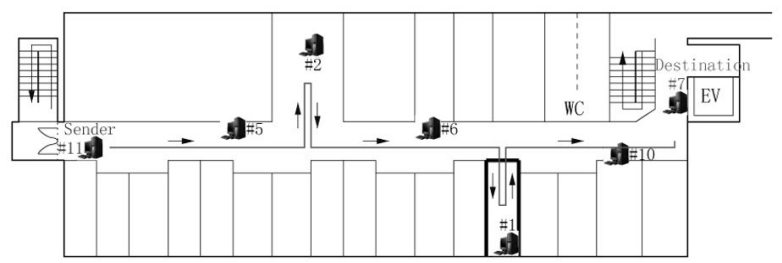

(c) DMS

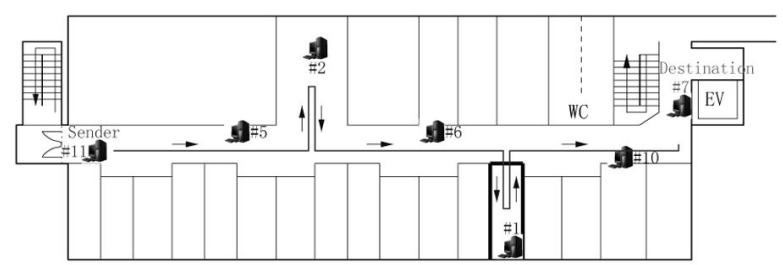

(b) SMS

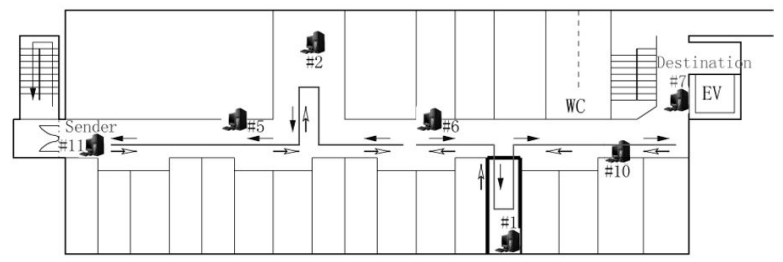

(d) SDMS

Fig. 3. Different topologies for experiments.

topologies for different experiments are shown in Fig. 3. All experimental parameters are shown in Table 2.

For the static scenario, 20 experiments were performed for each protocol, and every experiment lasted 60 seconds. The source node sent 512-byte packets, with a frequency of 200 packets per second. For the moving scenarios, we performed 10 experiments with a duration of 120 seconds each.

\subsection{Static scenario}

In the Static Scenario (SS), first all nodes are put in the positions shown in Fig. 3(a). Then, in each machine, the routing protocol deamons are started. In this paper, we consider AODV and OLSR and their deamons aodvd and olsrd, respectively. To let the routing protocol initialize routes, no data was transmitted for the first five minutes.

\subsection{Source moving scenario}

The Source Moving Scenario (SMS) is shown in Fig. 3(b). The nodes are in the same position as in SS (Fig. 3(a)), except that source node moved towards the destination node, as shown in Fig. 3(b). This movement is realized using a simple wheeled office chair.

\subsection{Destination moving scenario}

In Fig. 3(c), we show the Destination Moving Scenario (DMS). The destination node moves away from the source, starting its movement in the same position as the source node. At the end of 120 seconds, destination node and source node have the maximum distance between them. 
Table 3

Average values for different experiments

\begin{tabular}{cccccl}
\hline Nr. & Scenario & Protocol & Bitrate & Delay & Packetloss \\
\hline 1 & SS & AODV & 819.1863 & 0.0032 & 0.000076 \\
2 & & OLSR & 819.1727 & 0.0036 & 0.000056 \\
3 & \multirow{2}{*}{ SMS } & AODV & 613.9733 & 1.5855 & 0.2942 \\
4 & & OLSR & 618.8715 & 1.6504 & 0.2532 \\
5 & \multirow{2}{*}{ MMS } & AODV & 720.2372 & 0.7445 & 0.1654 \\
6 & & OLSR & 719.2644 & 0.8486 & 0.1597 \\
7 & \multirow{2}{*}{ SDMS } & AODV & 727.7739 & 0.8986 & 0.2265 \\
8 & & OLSR & 775.7824 & 0.8352 & 0.1656 \\
\hline
\end{tabular}

\subsection{Source-destination moving scenario}

As shown in Fig. 3(d), in Source-Destination Moving Scenario (SDMS), both source node and destination nodes are moving. Starting near the position of node 6 , they both move away from each other for the first 60 seconds. Then they go back by the same route, to the starting position for the last 60 seconds.

\section{Performance evaluation}

\subsection{Experimental settings}

We performed the experiments in indoor environment (our departmental floor), as shown in Fig. 3, with the size nearly $70 \mathrm{~m} \times 25 \mathrm{~m}$. We used UDP traffic for experimental environment (see Table 2 ). The D-ITG is used to create the traffic and to collect the data. Data in the network were collected in a Mesh Topology for different scenarios of node movement and for two routing protocols. We were interested in Bitrate (kbps), Delay (ms) and Packetloss (No.of packets).

We used CBR (Constant Bit Rate) over UDP to create the traffic. The transmission rate of the data flow is $200 \mathrm{pkts} / \mathrm{s}$, and the packet size is fixed to $512 \mathrm{kB}$, meaning a maximum bitrate of $819.2 \mathrm{kbps}$. Nodes (laptops) could access each other within the 70 meter region where the experiments were performed. We checked this by the ping command of Ubuntu 9.04. In total, we performed 8 experiments, as shown in Table 3.

As MAC protocol we used the IEEE $802.11 \mathrm{~b}$ protocol and configured the wireless cards to operate at central frequency $2.412 \mathrm{GHz}$ (channel 1) and with enough power to have connectivity with every node in the network. The main interest on these experiments was in the routing protocols and their behaviour in different scenarios, so all MAC parameters were kept unchanged. We should mention that during experiments all the IEEE 802.11 spectrum had been used by other access points operating within the campus, causing a considerable interference.

We took samples of $500 \mathrm{~ms}$ for every experiment, and computed the averages of each sample, using linux bash scripting and Matlab.

\subsection{Experimental measurements}

In Table 3, we show all the calculated average values for every experiment. We investigated all mean values of Bitrate, Delay and Packetloss, which are measured in "kilobits per second (kbps)", "milliseconds (ms)" and "percentage (\%)", respectively. 


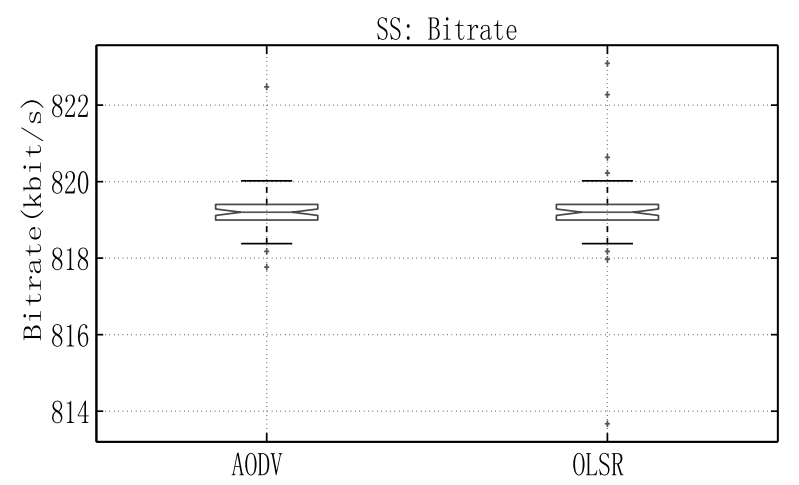

(a) Bitrate (boxplot)

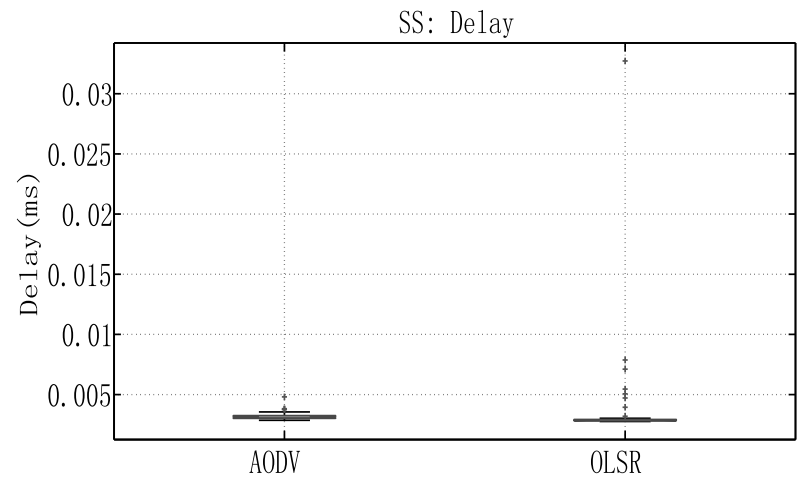

(b) Delay (boxplot)

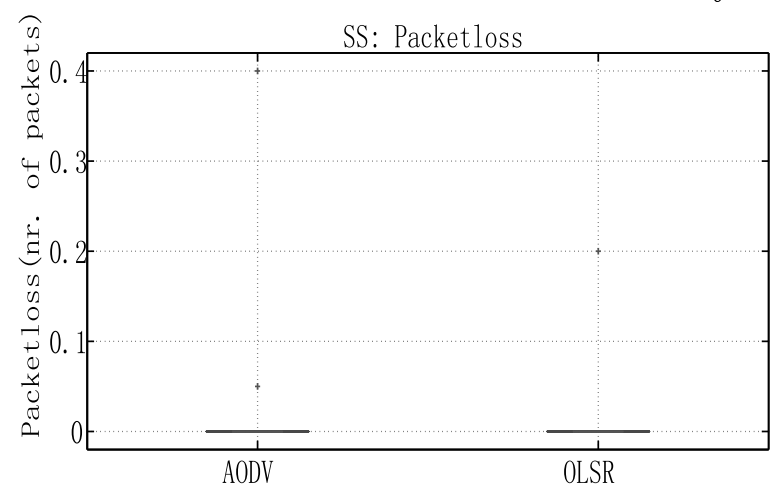

(c) Packetloss (boxplot)

Fig. 4. Different metrics vs different protocols for SS (boxplot).

For SS, in Fig. 4, we can see that for both AODV and OLSR, bitrate is almost the maximum (max = 819.2). This means that the routes have been established and the communication is performed at almost maximum performance. This is also shown in Table 3.

In SMS, the source node is approaching the destination node and at two time periods $30 \mathrm{~s}-50 \mathrm{~s}$ and 70 s-90 s they loose LOS (Line of Sight). In Figs 5 and 6, we show three metrics in time-domain and boxplot, respectively. In Fig. 5(a), the bitrate in the period of time $30 \mathrm{~s}-50 \mathrm{~s}$ reaches the value 0 . This means that the source node could not find a route to the destination node. At this period of time the nodes loose LOS and a complete route ( 2 or more hops) is difficult to be established. At the time period $70 \mathrm{~s}-90 \mathrm{~s}$, we also observe a decrease on the value of bitrate, which is more considerable in the case of AODV. In this case, even though there is no LOS between the communicating nodes, they are closer to each other and 1-hop or 2-hops routes can be quickly re-established.

In Fig. 6(a), we can observe that both protocols show the same performance regarding bitrate metric. At the period of time $30 \mathrm{~s}-50 \mathrm{~s}$, when the bitrate reaches very low values, we notice a proportional increase in packetloss as shown in Fig. 5(c). At time period $70 \mathrm{~s}-90 \mathrm{~s}$, we encountered a considerable amount of packet loss for AODV.

In Fig.6(c), it is shown that both protocols show almost the same performance considering packetloss metric. At time periods $30 \mathrm{~s}-50 \mathrm{~s}$, in the case of OLSR, we notice that the delay is increased as shown in Fig. 5(b). At this time period, the communicating nodes are in NLOS (Non Line of Sight) conditions and the communication needs 2 or more hops to occur. Thus, as described in [2], OLSR performance 


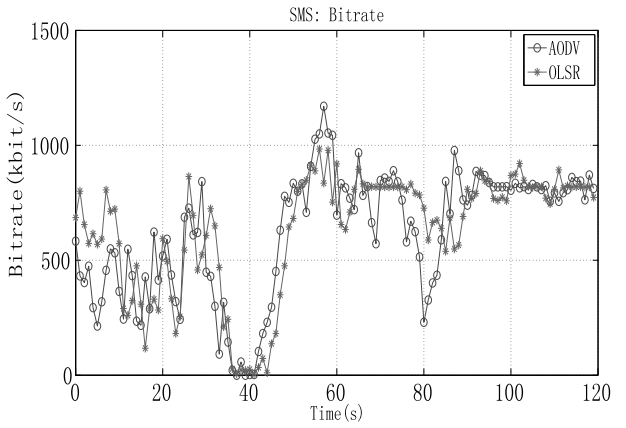

(a) Bitrate (time)

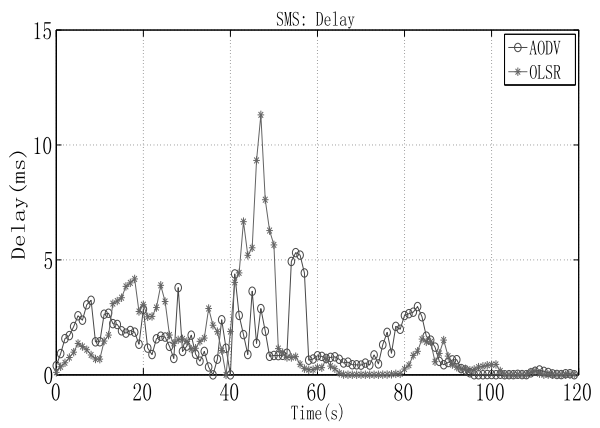

(b) Delay (time)

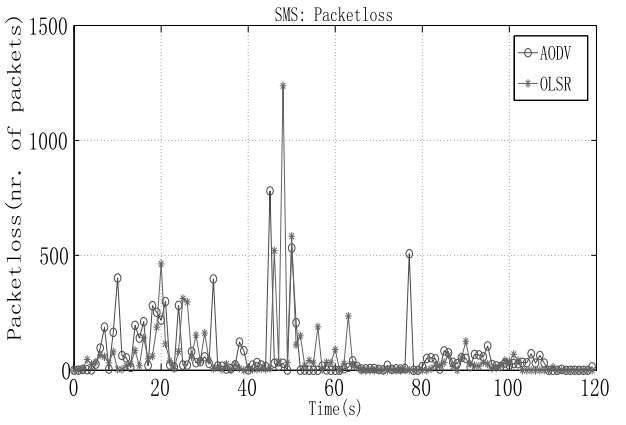

(c) Packetloss (time)

Fig. 5. Different metrics vs different protocols for SMS (time).

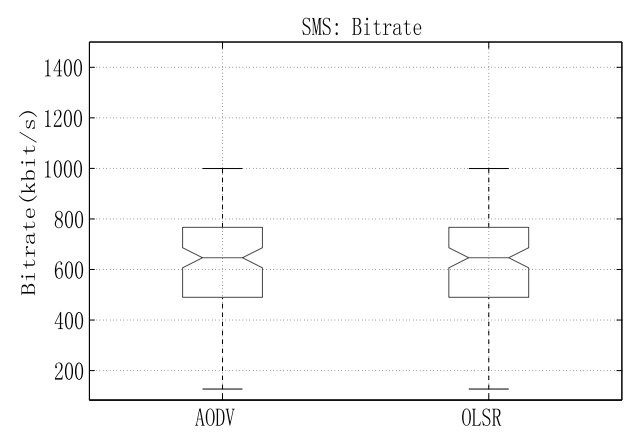

(a) Bitrate (boxplot)

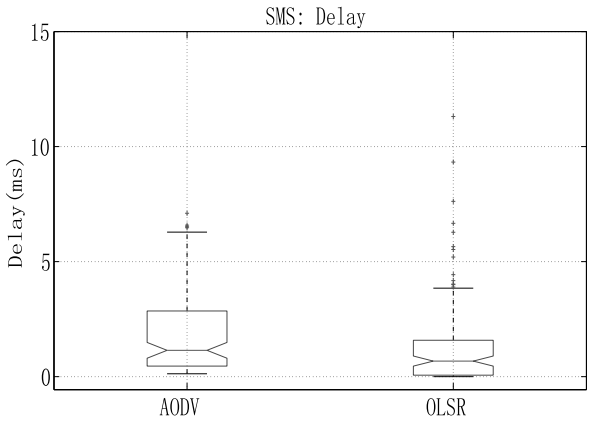

(b) Delay (boxplot)

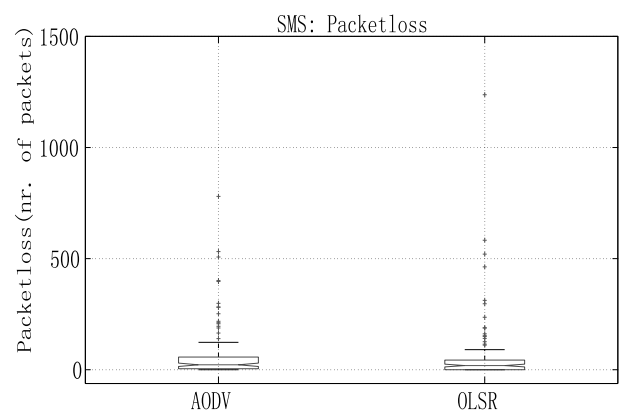

(c) Packetloss (boxplot)

Fig. 6. Different metrics vs different protocols for SMS (boxplot). 


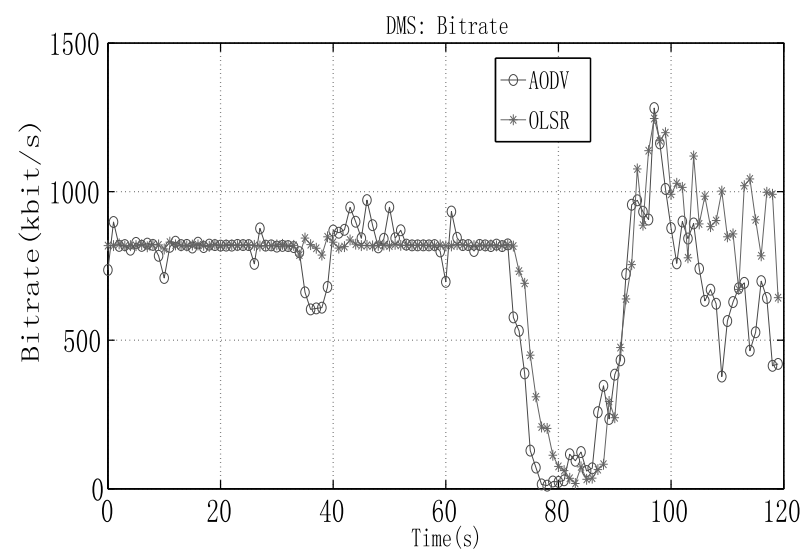

(a) Bitrate (time)

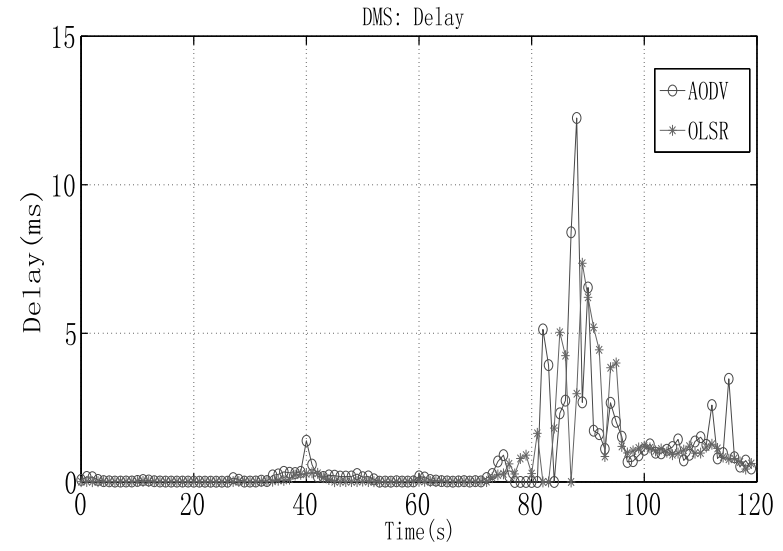

(b) Delay (time)

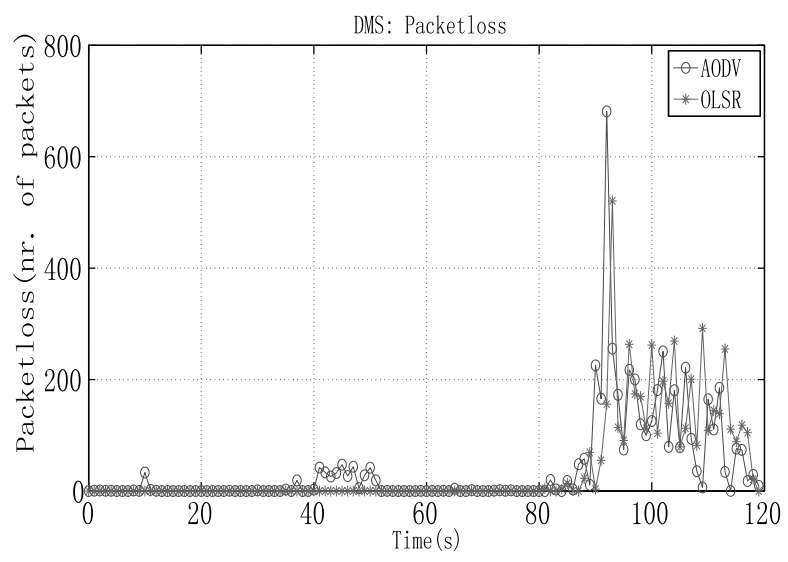

(c) Packetloss (time)

Fig. 7. Different metrics vs different protocols for DMS (time).

at 2-hops or 3-hops communication undergoes a degradation. As shown in Fig. 6(b), both OLSR and AODV protocols show the same performance considering the delay parameter.

In DMS, the destination node is moving away from the source node. In Figs 7 and 8, we show three metrics in time-domain and boxplot, respectively. In Fig. 7(a), the bitrate in the time period $75 \mathrm{~s}-90 \mathrm{~s}$ reaches the value 0 , which means the source node could not find a route to the destination node. At this period of time the two nodes loose LOS and a complete route of 2 or more hops is difficult to be established. As is shown in Fig. 8(a), the OLSR has a better throughput than AODV. After time $90 \mathrm{~s}$ the bitrate in case of AODV is lower than the case when OLSR is used. This happens because at that time, routes need to be re-established, and for AODV the route discovery process is not always successful, thus it needs more time. This fact is reflected in delay and packetloss graphs, respectively in Figs 7(b) and 7 (c) after time $90 \mathrm{~s}$. At the period of time $75 \mathrm{~s}-90 \mathrm{~s}$, when the bitrate reaches very low values, we notice a proportional increase in packetloss as shown in Fig. 7(c). After time $90 \mathrm{~s}$ the communications still has a considered amount of packetloss. In Fig. 8(c) is shown that AODV has a slightly worse performance than OLSR. At time period $75 \mathrm{~s}-90 \mathrm{~s}$, we notice an increased delay in Fig.7(b), which is due to the low bitrate experienced at these time periods. As shown in Fig. 8(b), both AODV and OLSR protocols have almost the same performance. 


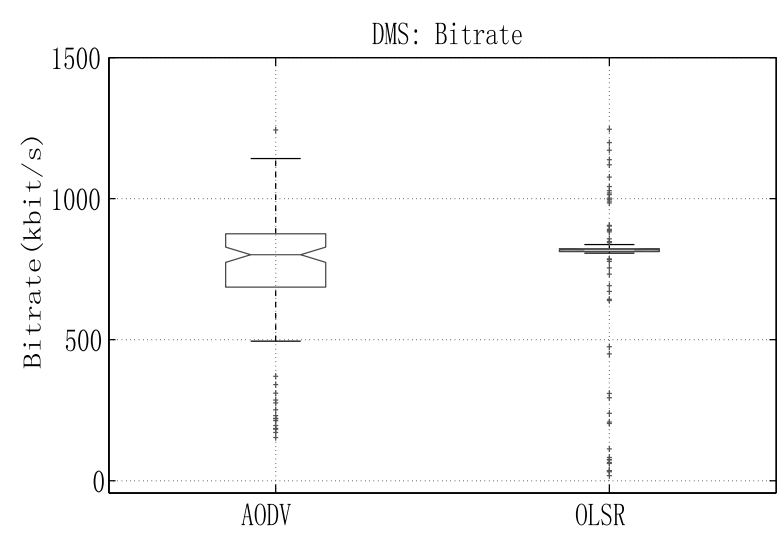

(a) Bitrate (boxplot)

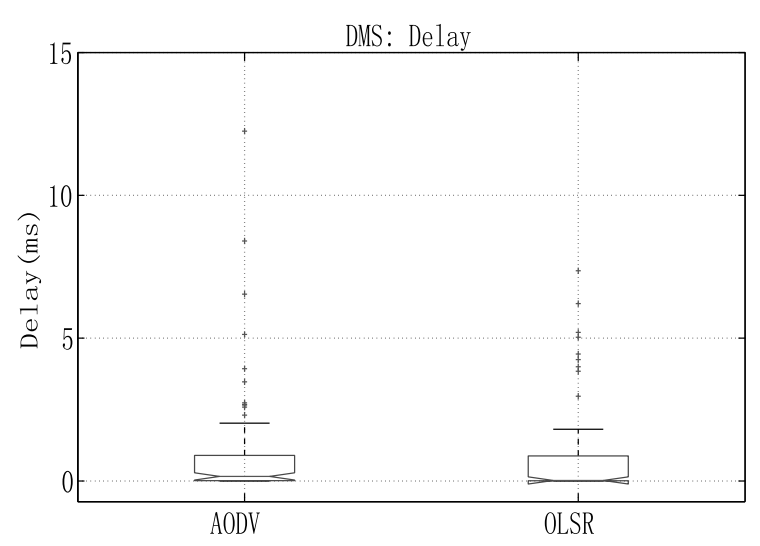

(b) Delay (boxplot)

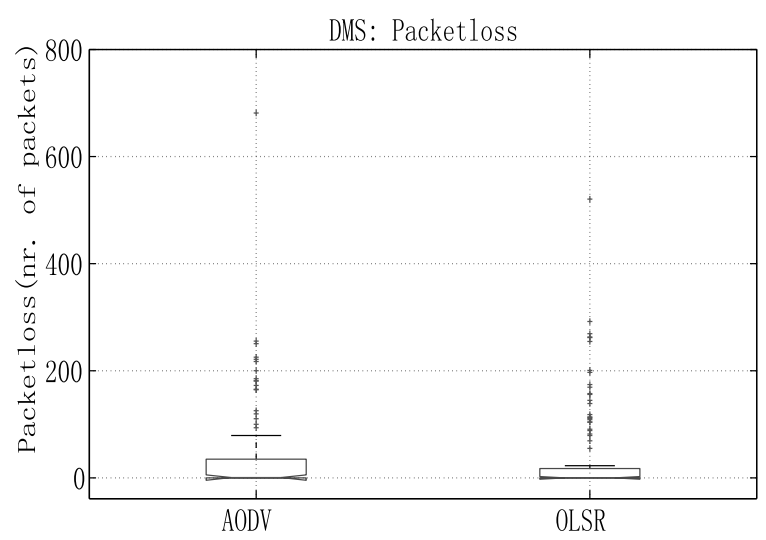

(c) Packetloss (boxplot)

Fig. 8. Different metrics vs different protocols for DMS (boxplot).

In SDMS during the first 60 seconds both nodes are moving away from each other and then during the last 60 seconds they are approaching each other via the same route of movement. In Figs 9 and 10, we show three metrics in time-domain and boxplot, respectively. In Fig. 9(a), the bitrate in the time periods $15 \mathrm{~s}-35 \mathrm{~s}$ and $90 \mathrm{~s}-105 \mathrm{~s}$ reaches the value 0 , which means the source node could not find a route to the destination node. At this periods of time the nodes loose LOS and a complete route of 2 or more hops is difficult to be established. As it is shown in Fig. 10(a), OLSR has a better performance than AODV regarding bitrate metric. At time periods $15 \mathrm{~s}-35 \mathrm{~s}$ and $90 \mathrm{~s}-105 \mathrm{~s}$ when bitrate reaches very low values, we notice a proportional increase in packetloss as shown in Fig. 9(c). In Fig. 10(c) for packetloss metric, AODV has a slightly worse performance than OLSR. At time periods $15 \mathrm{~s}-35 \mathrm{~s}$ and $90 \mathrm{~s}-105 \mathrm{~s}$, we notice an increased delay in Fig. 9(b), which is due to the low bitrate experienced at these time periods. As shown in Fig. 10(b), OLSR shows a better performance than AODV considering delay. This delay is caused by the continuous change of routes in SDMS.

AODV is more sensible to route changing, because it has to redefine the whole route before starting to send data. AODV protocol acts worse than OLSR in the cases when routes are lost. Being a reactive protocol, AODV has to redefine the communicating route, so it takes more time to re-establish the communication. In contrary OLSR chooses one of the old available routes, until the new routes are defined. 


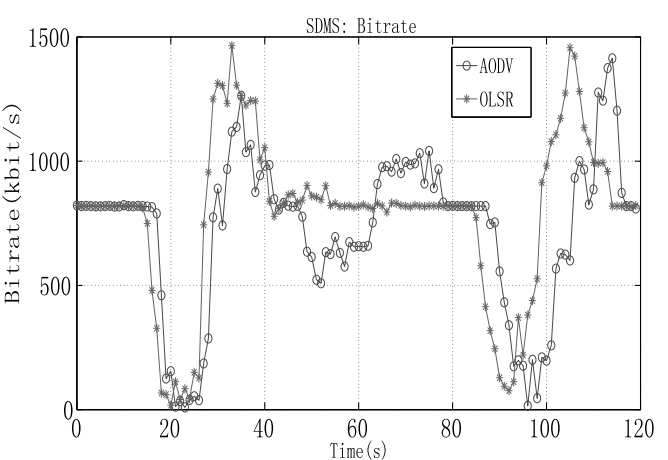

(a) Bitrate (time)

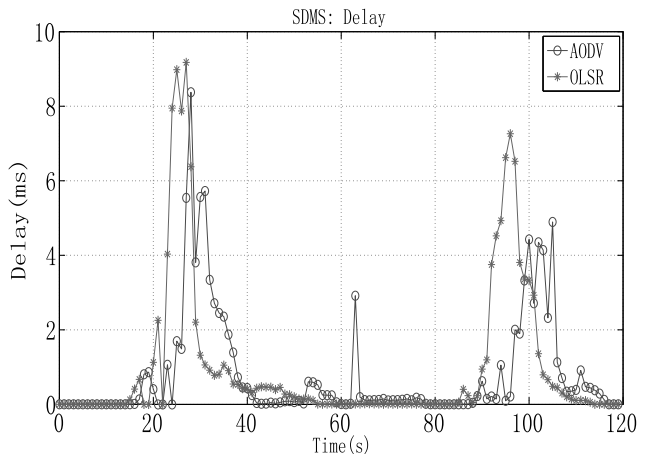

(b) Delay (time)

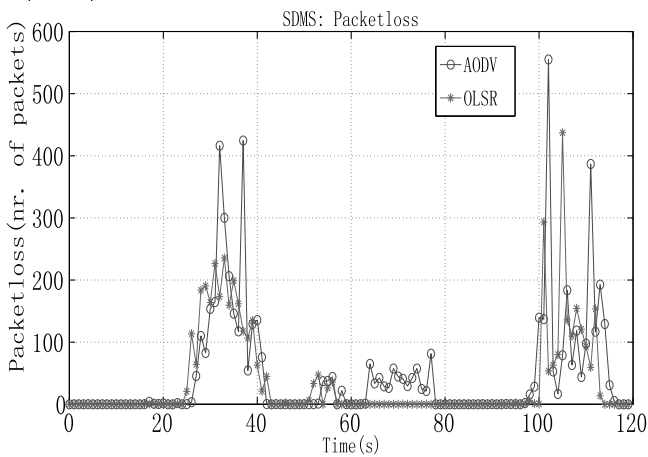

(c) Packetloss (time)

Fig. 9. Different metrics vs different protocols for SDMS (time).

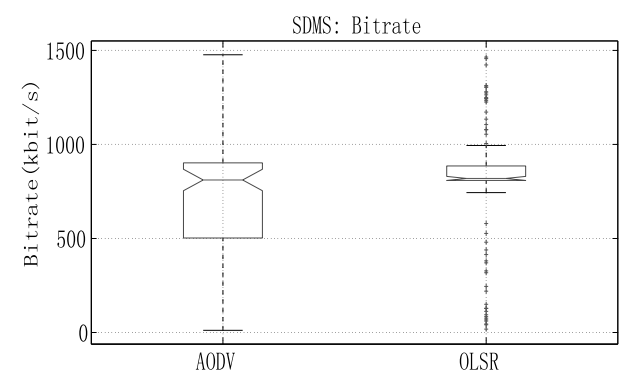

(a) Bitrate (boxplot)

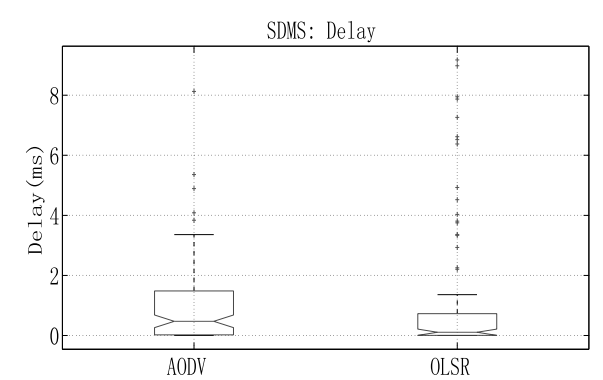

(b) Delay (boxplot)

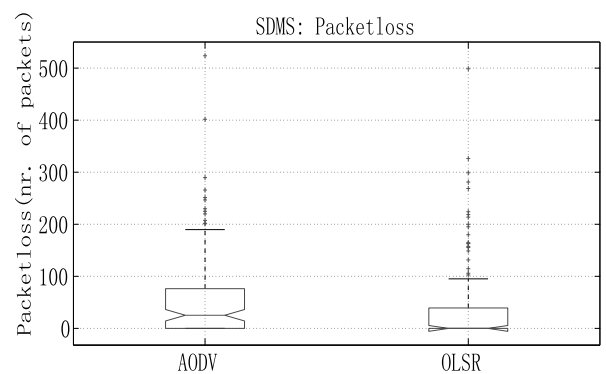

(c) Packetloss (boxplot)

Fig. 10. Different metrics vs different protocols for SDMS (boxplot). 


\section{Conclusions}

In this paper, we used AODV and OLSR protocols for experimental evaluation and comparison and we implemented four scenarios (SS, SMS, DMS and SDMS) in a small MANET testbed of 7 nodes. We considered 3 metrics for performance evaluation: bitrate, delay and packetloss. We investigated the performance of MANET when two communicating nodes loose LOS during a period of time.

From our experimental results we found that, when the communicating nodes are moving and the routes change quickly, OLSR as a proactive protocol performs better than AODV, which is a reactive protocol.

In our future work, we would like to increase the number of nodes in our testbed and implement more realistic moving scenarios. We will run multiple flows between the communicating nodes and we will use the linear topology, in order to minimize the interference caused by multiple links in mesh topology.

\section{Acknowledgement}

This work is support by a Grant-in-Aid for scientific research of Japanese Society for the Promotion of Science (JSPS). The authors would like to thank JSPS for the financial support.

\section{References}

[1] A. Aikebaier, T. Enokido and M. Takizawa, Design and Evaluation of Reliable Data Transmission Protocol in Wireless Sensor Networks, Mobile Information Systems 4(3) (2008), 237-252.

[2] L. Barolli, M. Ikeda, G. De Marco, A. Durresi and F. Xhafa, Performance Analysis of OLSR and BATMAN Protocols Considering Link Quality Parameter, Proc of IEEE AINA-2009 (2009), 307-314.

[3] T. Clausen and P. Jacquet, Optimized Link State Routing Protocol, Project Hipercom, INRIA Rocquencourt, France, Technical Report, RFC 3626 (Experimental), (2003) Available: http://hipercom.inria.fr/olsr/draft-ietf-manet-olsr-11.txt.

[4] Core Software, AODV Software, Available on line at http://core.it.uu.se/core/index.php/ AODV-UU.

[5] A. Durresi, P. Zhang, M. Durresi and L. Barolli, Architecture for Mobile Heterogeneous Multi Domain Networks, Mobile Information Systems 6(1) (2010), 49-63.

[6] D.S.J. De Couto, D. Aguayo, J. Bicket and R. Morris, A High Throughput Path Metric for Multi-hop Wireless Routing, Proc of MobiCom-2003, 9th Annual International Conference on Mobile Computing and Networking, (2003), 134-146, Available on line at http://dx.doi.org/10.1145/938985.939000.

[7] A. Dainotti, A. Botta and A. Pescap'e, Do You Know What You Are Generating?, Proc of ACM CoNEXT-2007 (2007), $1-2$.

[8] J. Goh and D. Taniar, Mining Frequency Pattern from Mobile Users, Proc of the 8th International Conference on Knowledge-Based Intelligent Information and Engineering Systems KES-2004, Part III, Lecture Notes in Computer Science, Springer, 3215 (2004), 795-801.

[9] J. Haemi, M. Fiore, F. Filali and C. Bonnet, A Realistic Mobility Simulator for Vehicular Ad Hoc Networks, EURECOM Technical Report, (2007), Available at: http://www.eurecom.fr/util/publidownload.en.htm?id=1811.

[10] A.M. Hanashi, I. Awan and M. Woodward, Performance Evaluation with Different Mobility Models for Dynamic Probabilistic Flooding in MANETs, Mobile Information Systems 5(1) (2009), 65-80.

[11] M. Ikeda, L. Barolli, M. Hiyama, G. De Marco, T. Yang and A. Durresi, Performance Evaluation of Link Quality Extension in Multihop Wireless Mobile Ad-hoc Networks, Proc of International Conference on Complex, Intelligent and Software Intensive Systems (CISIS-2009) (2009), 311-318.

[12] W. Kiess and M. Mauve, A Survey on Real-world Implementations of Mobile Ad-hoc Networks, Ad Hoc Networks 5(3) (2007), 324-339.

[13] V. Kawadia and P. Kumar, Experimental Investigations into TCP Performance over Wireless Multihop Networks, Proc of E-WIND-2005, ACM SIGCOMM workshop on Experimental Approaches to Wireless Network Design and Analysis (2005), 29-34.

[14] H. Lundgren, E. Nordstrom and C. Tschudin, Coping with Communication Gray Zones in IEEE 802.11b Based Ad hoc Networks, Proc of WoWMoM-2002 (2002), 49-55. 
[15] P. Larbig, RaLink RT2570 USB Enhanced Driver, Available on line at http://homepages.tu-darmstadt.de/ p_larbig/wlan/.

[16] Gj. Mino, L. Barolli, F. Xhafa, A. Durresi and A. Koyama, Implementation and Performance Evaluation of Two Fuzzybased Handover Systems for Wireless Cellular Networks, Mobile Information Systems 5(4) (2009), 339-361.

[17] S.S. Manvi, M.S. Kakkasageri and J. Pitt, Multiagent Based Information Dissemination in Vehicular Ad Hoc Networks, Mobile Information Systems 5(4) (2009), 363-389.

[18] OLSR Download, Available on line at http://www.olsr.org/.

[19] C. Perkins, E. Belding-Royer and S. Das, Ad hoc On-Demand Distance Vector (AODV) Routing, RFC3561, Nokia Research Center, University of California, University of Cincinnati, Technical Report, (2003) Available on line at http://www.ietf.org/rfc/rfc3561.txt.

[20] M. Piorkowski, M. Raya, A.L. Lugo, M. Grossglauser and J.P. Hubaux, Joint Traffic and Network Simulator for VANETs, Proc of Mobile and Information Communication Systems Conference (MICS-2006), (October 2006) Available on line at: http://www.mics.ch/.

[21] V. Pham, E. Larsen, O. Kure and P. Engelstad, Routing of Internal MANET Traffic over External Networks, Mobile Information Systems 5(3) (2009), 291-311.

[22] The Network Simulator NS-2, Available on line at http://www.isi.edu/nsnam/.

[23] C. Tschudin, P. Gunningberg, H. Lundgren and E. Nordstrom, Lessons from Experimental MANET Research, Ad Hoc Networks 3(2) (2005), 221-233.

[24] D. Taniar and J. Goh, On Mining Movement Pattern from Mobile Users, International Journal of Distributed Sensor Networks 3(1) (2007), 69-86.

[25] K. Xuan, G. Zhao, D. Taniar and B. Srinivasan, Continuous Range Search Query Processing in Mobile Navigation, Proc of IEEE ICPADS-2008 (2008), 361-368.

[26] T. Yang, G. De Marco, M. Ikeda and L. Barolli, Impact of Radio Randomness on Performances of Lattice Wireless Sensors Networks Based on Event-reliability Concept, Mobile Information Systems 2(4) (2006), 211-227.

Elis Kulla received his B.S and M.S degrees at Faculty of Information Technology, Polytechnic University of Tirana (PUT), Albania in 2007 and 2010, respectively. Presently, he is a Ph.D Student at Graduate School of Engineering, Fukuoka Institute of Technology (FIT), Japan. His research interests include ad-hoc networks, sensor networks, P2P networks, and vehicular networks.

Masahiro Hiyama received his B.S degree at Fukuoka Institute of Technology (FIT), Japan in 2009. Presently, he is a Master Student at Graduate School of Engineering, FIT, Japan. His research interests include ad-hoc networks and sensor networks.

Makoto Ikeda is an Assistant Research Fellow at the Center for Asian and Pacific Studies, Seikei University, Japan. He received B.S, M.S and Ph.D degrees in Information and Communication Engineering from Fukuoka Institute of Technology (FIT), Japan, in 2005, 2007, and 2010, respectively. He has published about 30 research papers in Journals and International Conference Proceedings. He won the Best Paper Award at NBiS-2008 International Conference. He has been a PC Member and Web Administrator for some International Conferences. His research interests include wireless networks, mobile computing, high-speed networks, P2P systems, ad-hoc networks and sensor networks. He is a member of the IEEE, ACM, IPSJ and IEICE.

Leonard Barolli received B.S and Ph.D degrees from Tirana University and Yamagata University in 1989 and 1997, respectively. From April 1997 to March 1999, he was a JSPS Post Doctor Fellow Researcher at Department of Electrical and Information Engineering, Yamagata University. From April 1999 to March 2002, he worked as a Research Associate at the Department of Public Policy and Social Studies, Yamagata University. From April 2002 to March 2003, he was an Assistant Professor at Department of Computer Science, Saitama Institute of Technology (SIT). From April 2003 to March 2005, he was an Associate Professor and presently is a Full Professor, at Department of Information and Communication Engineering, Fukuoka Institute of Technology (FIT). Dr. Barolli has published about 300 papers in referred Journals, Books and International Conference proceedings. He was an Editor of the IPSJ Journal and has served as a Guest Editor for many International Journals. Dr. Barolli has been a PC Member of many International Conferences and was the PC Chair of IEEE AINA-2004 and IEEE ICPADS2005. He was General Co-Chair of IEEE AINA-2006, AINA-2008, AINA-2010, CISIS-2009 and CISIS-2010, Workshops Chair of iiWAS-2006/MoMM-2006 and iiWAS-2007/MoMM-2007, Workshop Co-Chair of ARES-2007, ARES-2008, IEEE AINA-2007 and ICPP-2009. Dr. Barolli is the Steering Committee Chair of CISIS and BWCCA International Conferences and is serving as Steering Committee Co-Chair of IEEE AINA, NBiS and 3PGCIC International Conferences. He is organizers of many International Workshops. Dr. Barolli has won many Awards for his scientific work and has received many research funds. He got the "Doctor Honoris Causa" Award from Polytechnic University of Tirana in 2009. His research interests include network traffic control, fuzzy control, genetic algorithms, agent-based systems, ad-hoc networks and sensor networks. He is a member of SOFT, IPSJ, and IEEE. 
Vladi Kolici received his B.S and M.S degrees in Telecommunication Engineering from Polytechnic University of Tirana (PUT) in 1997 and 2005, respectively. He obtained his Ph.D from PUT in May 2009. From 1997 to 2004, he was a Research Associate and from 2005 to present he is a Lecturer at Department of Electronics and Telecommunications, Faculty of Information Technology, PUT. He is teaching several courses in the areas of wireless and mobile networking, P2P systems and quality of services. Dr. Kolici has published several papers in International and National Conference Proceedings in the areas of P2P and Ad-Hoc networks. Dr. Kolici received the Best Application Paper Award at the 6th International Conference on Advances in Mobile Computing and Multimedia (MoMM-2008) in 2008, Linz, Austria. His research interests include P2P networks, wireless and mobile networks, and high speed networks.

Rozeta Miho received her B.S and M.S degrees in Electronic and Telecommunication Engineering from Polytechnic University of Tirana (PUT), Albania, in 1985 and 1989, respectively. She obtained her Ph.D from PUT in November 1995. From 1985 to 1995, she was a lecturer, from 1996 to 2001 Assistant Professor, from 2001 to 2007 Associate Professor, and presently she is a Full Professor of PUT. From May 2009, she is the Dean of Faculty of Information Technology, PUT. She is teaching several courses in the areas of telecommunication networks, optical communications and optical fibre networks. Prof. Miho has published several papers in International and National Conference Proceedings in the areas of optical WDM networks, P2P and Ad-Hoc networks. She is the co-author of the Best Application Paper Award at the 6th International Conference on Advances in Mobile Computing and Multimedia (MoMM-2008) in 2008, Linz, Austria. Her research interests include P2P networks, optical networks, wireless networks and high speed networks. 

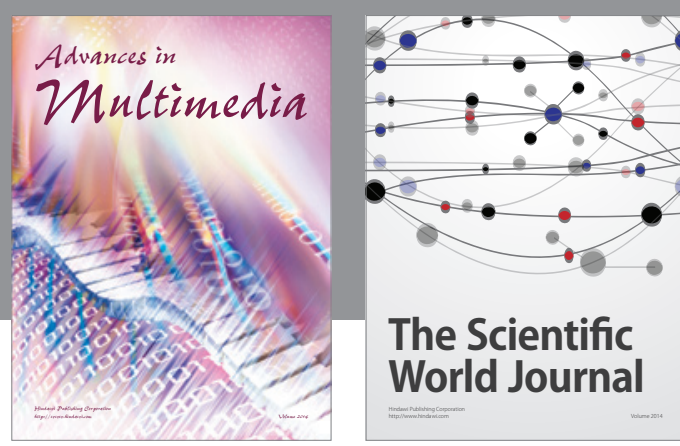

The Scientific World Journal
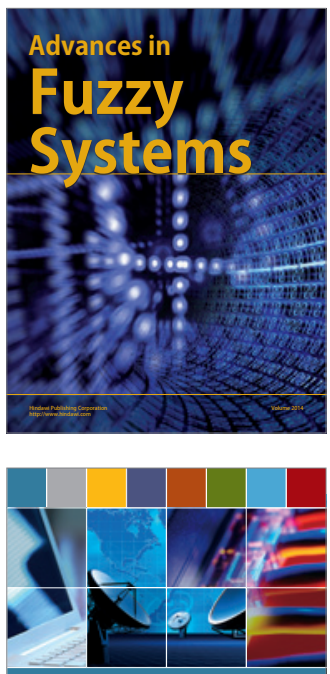

Computer Networks and Communications
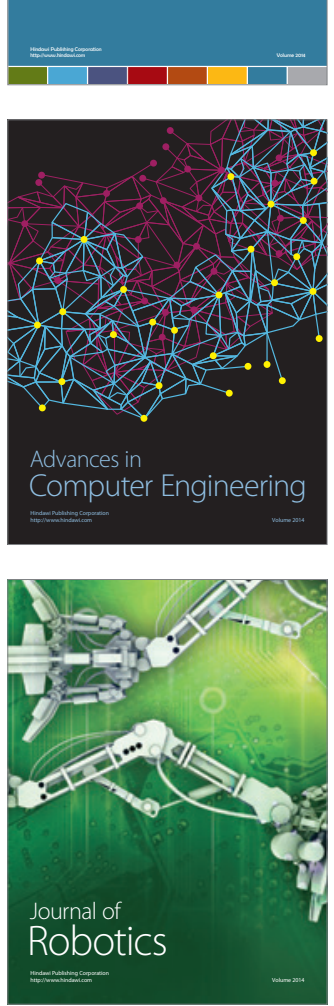
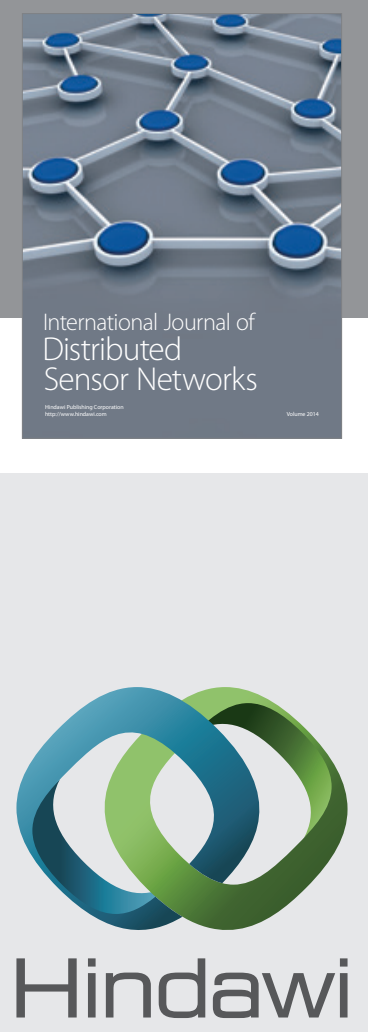

Submit your manuscripts at

http://www.hindawi.com
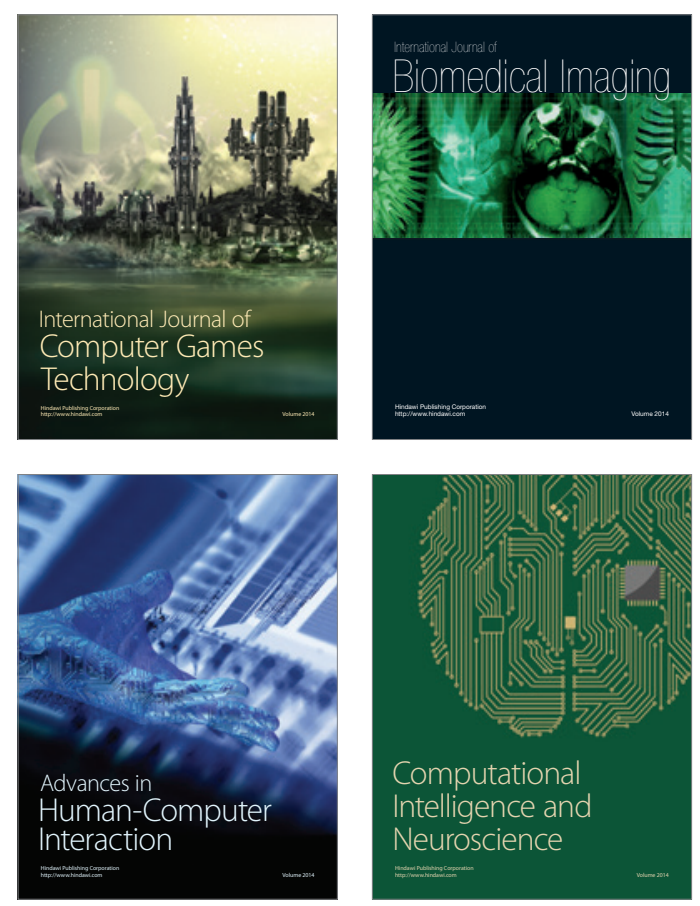
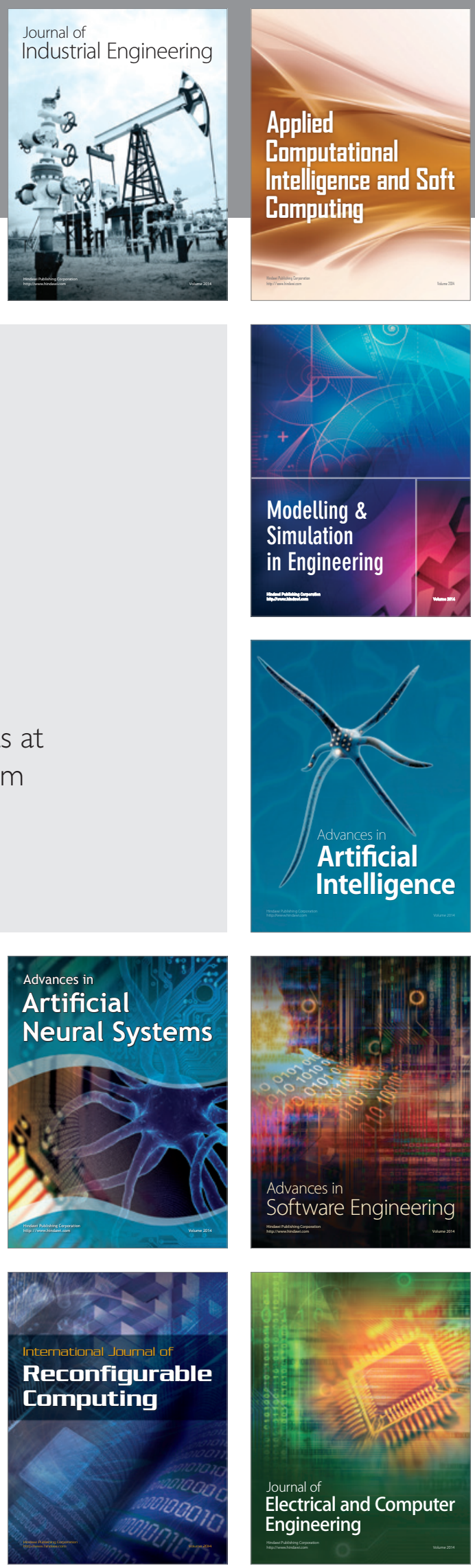